\title{
Degree-constrained orientations of embedded graphs
}

\author{
Yann Disser ${ }^{1}$ and Jannik Matuschke ${ }^{2}$ \\ 1 ETH Zurich, Intitute of Theoretical Computer Science, ydisser@inf .ethz.ch \\ ${ }^{2}$ TU Berlin, Institut für Mathematik, matuschke@math.tu-berlin.de
}

\begin{abstract}
We investigate the problem of orienting the edges of an embedded graph in such a way that the in-degrees of both the nodes and faces meet given values. We show that the number of feasible solutions is bounded by $2^{2 g}$, where $g$ is the genus of the embedding, and all solutions can be determined within time $\mathcal{O}\left(2^{2 g}|E|^{2}+|E|^{3}\right)$. In particular, for planar graphs the solution is unique if it exists, and in general the problem of finding a feasible orientation is fixed-parameter tractable in $g$. In sharp contrast to these results, we show that the problem becomes NP-complete even for a fixed genus if only upper and lower bounds on the in-degrees are specified instead of exact values.
\end{abstract}

\section{Introduction}

Graph orientation is an area of combinatorial optimization that deals with the problem of assigning directions to the edges of an undirected graph, subject to certain problem-specific requirements. Besides yielding useful structural insights, e.g., with respect to connectivity of graphs [14] and hypergraphs [11], research in graph orientation is motivated by applications in areas such as graph drawing $[2$, $5]$ or efficient data structures for planar graphs [3].

A particularly well-studied class of orientation problems are degree-constrained problems, i.e., where the in-degree of each vertex in the resulting orientation has to lie within certain bounds. Hakimi [12] and Frank [10] provided good characterizations $^{3}$ for the existence of such orientations. In this paper, we answer a question raised by András Frank [9], asking for a good characterization for the following problem: Given an embedding of a graph in the plane, is there an orientation of the edges that meets prescribed in-degrees both in the primal and the dual graph at the same time? We show that if such an orientation exists, it is unique and can be computed by combining a feasible orientation for the primal graph with a feasible orientation for the dual graph. Our result generalizes to graph embeddings of higher genus, showing that the number of feasible orientations is bounded by a function of the genus, and the set of all solutions can be computed efficiently as long as the genus is fixed. We also show

\footnotetext{
${ }^{3}$ A good characterization of a decision problem in the sense of Edmonds [6] is a description of polynomially verifiable certificates for both yes- and no-instances of the problem.
} 
that the problem becomes NP-complete as soon as upper and lower bounds on the in-degrees are specified instead of exact values.

Related work. Research in graph orientation has a long history that revealed many interesting structural insights and applications. E.g., a classical result by Robbins [14] states that an undirected graph is 2-edge-connected if and only if it has an orientation that is strongly connected. This result was translated to hypergraphs by Frank et al. [11]. Graph orientation is also closely connected to graph drawing. For example, Eades and Wormald [5] showed hardness of a fixed edge-length graph drawing problem using an orientation problem on a planar graph as an important device in their reduction. More recently, Biedl et al. [2] provided a 13/8-approximation algorithm for finding a balanced acyclic orientation, with implications for orthogonal graph drawing.

Regarding degree-constrained orientation problems, Hakimi [12] gave a good characterization for the existence of orientations that match given prescribed in-degrees exactly and also for the existence of orientations that fulfill either lower or upper bounds on the in-degrees. Frank and Gyárfás [10] observed that the results for lower and upper bounds can easily be combined in a constructive way to find orientations that fulfill upper and lower bounds at the same time. Asahiro et al. [1] consider an optimization version of the degree-constrained orientation problem where a penalty function on the violated degree-bounds is to be minimized. They find that the problem is solvable in polynomial time if the penalty function is convex, but APX-hard in case of concave penalty functions.

Orientations of planar graphs received special attention by the research community because they revealed several interesting properties. Based on the insight that every planar graph allows for an orientation with maximum in-degree 3 , Chrobak and Eppstein [3] designed a highly efficient data structure for adjacency queries in planar graphs. In a distinct line of research, Felsner [7] showed that the set of orientations fulfilling a prescribed in-degree in a planar graph carries the structure of a distributive lattice.

Contribution and structure of the paper. In this paper, we consider an extension of the degree-constrained problem, which we call primal-dual orientation problem. The input to this problem is an embedding of a graph in a surface and we require in-degree prescriptions not only to be met for every vertex but also for every face of the embedding (in this context, the in-degree of a face refers to the number of edges on its boundary oriented in counter-clockwise direction). This variant of the problem was first proposed by András Frank for the special case of plane graphs [9] in conjunction with the question for a good characterization of the existence of such an orientation.

Before we present our results, we give a short introduction to orientations and embedded graphs in Section 2. Section 3 then deals with the primal-dual orientation problem with fixed in-degrees and contains two different proofs that yield the answer to Frank's question. Subsection 3.1 comprises a combinatorial proof for the uniqueness of the solution in plane graphs, also reducing the problem to solving the original degree-constrained orientation problem once in the 
primal and once in the dual graph. In Subsection 3.2, an alternative proof based on a simple linear algebra argument also yields a bound on the number of feasible orientations in embeddings of higher genus - showing that the problem is fixed-parameter tractable in terms of the genus. In Section 4, we show that if we accept bounds on the in-degrees instead of exact values, the problem becomes NP-complete. In Section 5, we point out an open question, which will be subject of future research.

\section{Preliminaries}

We give a short introduction on graph embeddings and orientations of those embeddings. Throughout this paper we will assume all graphs to be connected but not necessarily simple, i.e., loops and multi-edges are allowed. While the connectedness assumption is very common in the context of graph embeddings, all results presented here can be extended to non-connected graphs by temporarily introducing additional edges (and adjusting the in-degree specifications accordingly) so as to render the graph connected.

Embedded graphs. An embedding of a graph is a mapping of its vertices and edges onto a closed surface (e.g., a sphere or a torus) such that edges meet only at common vertices. This mapping partitions the surface into several regions, called faces. The dual of an embedded graph is the graph that is obtained by the following procedure: For every face in the embedding, introduce a vertex in the dual graph. For every edge of primal graph, introduce an edge in the dual graph that connects the faces that are adjacent to the original edge. The genus $g$ of the embedding is determined by Euler's formula: If $E$ is the set of edges, $V$ is the set of vertices and $V^{*}$ is the set of faces, then $|V|+\left|V^{*}\right|-|E|=2-2 g$.

If $g=0$, i.e., the graph is embedded in a sphere, the embedding is called planar (as embeddings in spheres and planes are combinatorially equivalent). Planar embeddings have several features that make them particularly interesting. In this work, we will make use of the following fact, called cycle-cut duality [15], which holds (exclusively) in planar embeddings: A set of edges is a simple cycle in the primal if and only if it is a simple cut ${ }^{4}$ in the dual and vice versa.

Orientations of primal and dual graphs. An orientation of a graph is an assignment of directions to the edges, i.e., for every edge we specify one of the two endpoints of the edge as its head and the other as its tail. By convention, we orient the edges in the dual graph in such a way that they cross their primal "alter egos" from right to left (cf. Figure 1). Thus every orientation of the primal graph induces an orientation of the dual graph and vice versa. Given an orientation $D$, we denote the set of edges whose head is the vertex $v$ by $\delta_{D}^{-}(v)$ and the set of edges whose tail is $v$ by $\delta_{D}^{+}(v)$. In accordance with our convention for dual orientations, we let $\delta_{D}^{-}(f)$ be the set of edges whose left face is $f$, and $\delta_{D}^{+}(f)$ be the set of edges whose right face is $f$.

\footnotetext{
${ }^{4}$ A simple cut is a cut whose edge set is minimal w.r.t. inclusion. In a connected graph, a cut is simple if and only if it splits the graph into two connected components.
} 


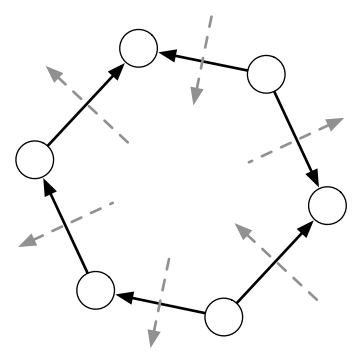

Fig. 1. Induced orientations of the edges in the dual graph. An edge in the dual graph crosses its corresponding edge in the primal graph from right to left.

We mention that our convention for primal and dual orientations extends cycle/cut duality in the sense that a directed simple cycle in the primal is a directed simple cut in the dual and vice versa. ${ }^{5}$

\section{Orientations with fixed in-degrees}

We consider the problem of finding an orientation that meets given fixed indegrees for both the vertices and faces of the embedded graph, called the primaldual orientation problem. We start this section by stating a formal description of the problem.

Problem 1. (Primal-dual orientation problem)

Given: an embedded graph $G=(V, E)$, two functions $\alpha: V \rightarrow \mathbb{N}_{0}, \alpha^{*}: V^{*} \rightarrow \mathbb{N}_{0}$

Task: Find an orientation $D$ of the edges $E$ such that $\left|\delta_{D}^{-}(v)\right|=\alpha(v)$ for all $v \in V$ and $\left|\delta_{D}^{-}(f)\right|=\alpha^{*}(f)$ for all $f \in V^{*}$, or prove that there is none.

Primal and dual feasibility. The following notation will be useful throughout the proofs in this section. Given an instance of the primal-dual orientation problem, we call an orientation $D$

- primally feasible if $\left|\delta_{D}^{-}(v)\right|=\alpha(v)$ for all $v \in V$.

- dually feasible if $\left|\delta_{D}^{-}(f)\right|=\alpha^{*}(f)$ for all $f \in V^{*}$.

- totally feasible if it is primally and dually feasible.

The primal-dual orientation problem thus asks for a totally feasible orientation. It is clear that the existence of both primally feasible solutions and dually feasible solutions is necessary for the existence of such a totally feasible orientation. However, it can easily be checked that this is not sufficient: For example, consider

\footnotetext{
${ }^{5}$ A cut or cycle is directed if all its edges are oriented in the same direction.
} 
a planar graph with two vertices and two parallel edges connecting them, and let $\alpha(v)=1$ and $\alpha^{*}(f)=1$ for all $v \in V$ and $f \in V^{*}$. While orienting both edges in opposite directions in the primal graph is primally feasible, orienting them in the same direction (which is orienting them in oposite directions in the dual graph) is dually feasible. However, none of the orientations is totally feasible.

In this section, we will present two approaches for obtaining necessary and sufficient conditions for the existence of totally feasible solutions.

\subsection{A combinatorial approach for planar embeddings}

In this section we want to provide a combinatorial argument for the uniqueness of a feasible solution to the primal-dual orientation problem in the planar case. We show how to construct a totally feasible solution from an orientation that is feasible in the primal graph and an orientation that is feasible in the dual graph.

Rigid edges. Hakimi [12] showed that a primally feasible orientation exists if and only if $\sum_{v \in V} \alpha(v)=|E|$ and $\sum_{v \in S} \alpha(v) \geq|E[S]|$ for all $S \subseteq V$, where $E[S]$ is the set of edges with both endpoints in $S$. The necessity follows from the fact that every edge in $E[S]$ contributes to the in-degree of a node in $S$, independent of its orientation.

Now consider a subset $S \subseteq V$ with $\sum_{v \in S} \alpha(v)=|E[S]|$. All edges that have one end point in $S$ and one end point in $V \backslash S$ must be oriented from $S$ to $V \backslash S$ in all primally feasible orientations. We call edges whose orientation is fixed in this way primally rigid ${ }^{6}$ and denote the set of all primally rigid edges by $R$. Analogously, we define the set of dually rigid edges $R^{*}$ as those that are fixed for all dually feasible orientations due to a tight set $S^{*} \subseteq V^{*}$ of faces with $\sum_{f \in S^{*}} \alpha^{*}(f)=\left|E\left[S^{*}\right]\right|$. It is easy to check that an edge is primally rigid if and only if it is on a directed cut in the primal graph with respect to any primally feasible orientation. Likewise, an edge is dually rigid if it is on a directed cut in the dual graph with respect to any dually feasible orientation. Furthermore, note that the set of edges on directed cuts is invariant for all feasible solutions.

Our main result in this section follows from this characterization of rigid edges and the duality of cycles and cuts in planar graphs.

Theorem 1. In case of a planar embedding, there exists a totally feasible orientation if and only if the following three conditions are fulfilled.

(1) There exists both a primally feasible orientation $D$ and a dually feasible orientation $D^{*}$.

(2) The edge set can be partitioned into primally and dually rigid edges $(E=$ $\left.R \dot{\cup} R^{*}\right)$.

(3) The orientation obtained by orienting all primally rigid edges in the same direction as they are oriented in $D$ and all dually rigid edges in the same orientation as they are oriented in $D^{*}$ is totally feasible.

If it exists, the solution is unique.

\footnotetext{
${ }^{6}$ The term "rigid" for edges on a directed cut of an orientation is taken from [7].
} 
Proof. The sufficiency of the conditions is trivial, as the third condition requires the existence of a totally feasible orientation. In order to show necessity, assume there exists a totally feasible orientation $D_{0}$. As $D_{0}$ is both primally and dually feasible, it fulfills Condition (1) of the theorem. An edge is primally rigid if and only if it is on a directed cut (w.r.t. $D_{0}$ ) in the primal graph. It is dually rigid, if and only if it is on a directed cut in the dual graph. Thus, by cycle/cut duality of planar graphs, an edge is dually rigid if and only if it is on a directed cycle in the primal graph. As every edge in the primal graph is either on a directed cut or on a directed cycle, the sets of primally and dually rigid edges comprise a partition of $E$, proving Condition (2). Now, let $D$ be a primally feasible orientation and $D^{*}$ be a dually feasible orientation. As $D_{0}$ equals $D$ on all primally rigid edges and equals $D^{*}$ on all dually rigid edges, the construction described in Condition (3) yields $D_{0}$ and is feasible. As all edges are either primally of dually rigid, they must have the same orientation in all totally feasible solutions, and $D_{0}$ is unique.

Note that the totally feasible solution constructed in the third condition does not depend on the choice of $D$ and $D^{*}$. Theorem 1 also yields a polynomial time algorithm to solve the problem for planar embeddings.

Corollary 2. The primal-dual orientation problem in planar embeddings can be solved in time $\mathcal{O}\left(|E|^{2}\right)$.

Proof. By Theorem 1, the problem can be solved by computing a primally feasible solution and a dually feasible solution and identifying the corresponding rigid edges. A primally feasible orientation can be found in time $\mathcal{O}(|V||E|)$ by using a simple push/relabel type algorithm [8]. Now applying the same result to the dual gives a total time of $\mathcal{O}\left(\left(|V|+\left|V^{*}\right|\right) \cdot|E|\right)=\mathcal{O}\left(|E|^{2}\right)$ for determining the two orientations. Identifying directed cuts is equivalent to identifying strongly connected components, which can be done in time $\mathcal{O}(|E|)$.

\subsection{A linear algebra analysis for general embeddings}

The primal-dual orientation problem can be formulated as a system of linear equalities over binary variables. To this end, we fix an arbitrary orientation $D$ of the graph and introduce for every edge $e \in E$ a decision variable $x(e)$ that determines whether the orientation of the edge should be reversed (if it is 1) or not (if it is 0 ) in order to become totally feasible. The vector $x \in\{0,1\}^{E}$ yields a feasible orientation if and only if it satisfies the following system of equalities:

$$
\begin{aligned}
& \sum_{e \in \delta_{D}^{+}(v)} x(e)-\sum_{e \in \delta_{D}^{-}(v)} x(e)=\alpha(v)-\left|\delta_{D}^{-}(v)\right| \quad \forall v \in V \\
& \sum_{e \in \delta_{D}^{+}(f)} x(e)-\sum_{e \in \delta_{D}^{-}(f)} x(e)=\alpha^{*}(f)-\left|\delta_{D}^{-}(f)\right| \forall f \in V^{*}
\end{aligned}
$$

The matrix corresponding to the first set of equalities is the incidence matrix of the primal graph, while the matrix corresponding to the second type of equalities is the incidence matrix of the dual graph (both graphs directed according 


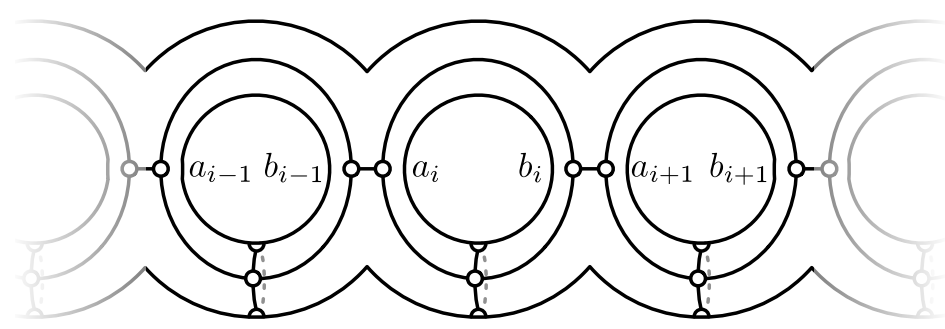

Fig. 2. Construction of an instance with $2^{2 g}$ feasible orientations, showing the tightness of the bound in Theorem 3 . The base graph consists of two cycles of length 3 intersecting in a common vertex and is embedded in a torus. Examples of genus $g$ are obtained by introducing $g$ copies of the base graph.

to the orientation $D$ ). As we assume the graph to be connected, we know that the rank of the former matrix is $|V|-1$, while the rank of the latter matrix is $\left|V^{*}\right|-1$. Using the fact that the boundary of a face is a closed walk in the primal graph, it is easy to see that the rows of the first matrix are orthogonal to the rows of the second matrix. This implies that all feasible solutions are contained in a subspace of $\mathbb{R}^{E}$ of dimension $|E|-|V|-\left|V^{*}\right|+2=2 g$.

Theorem 3. There are at most $2^{2 g}$ distinct solutions to the primal-dual orientation problem. The set of all totally feasible orientations can be determined in time $\mathcal{O}\left(2^{2 g}|E|^{2}+|E|^{3}\right)$. The bound on the number of orientations is tight, i.e., there are embedded graphs of genus $g$ that allow for $2^{2 g}$ distinct orientations.

Proof. By basis augmentation, there is a set $A \subseteq E$ of $2 g$ edges such that adding equalities $x(e)=a(e)$ with $a(e) \in\{0,1\}$ for all $e \in A$ results in a system with full rank, i.e., it has at most one solution. If for some $a \in\{0,1\}^{A}$ the unique solution exists and is a 0 -1-vector, it corresponds to the unique totally feasible orientation that orients the edges of $A$ according to the values $a(e)$. Otherwise, there is no such totally feasible orientation. Thus, solving the equality system for all $\left|\{0,1\}^{A}\right|=2^{2 g}$ possible values of $a$ yields all possible solutions to the primaldual orientation problem. This takes time $\mathcal{O}\left(|E|^{3}\right)$ for inverting the $|E| \times|E|$ matrix and $\mathcal{O}\left(2^{2 g}|E|^{2}\right)$ for multiplying the $2^{2 g}$ distinct right hand side vectors.

To see that the bound on the number of orientations is tight, consider the example depicted in Figure 2. It is constructed from a base graph consisting of two cycles of length 3 sharing a common vertex. The base graph is embedded in a torus, thus featuring only a single face $f$. When setting $\alpha^{*}(f)=|E|=6$, any orientation is dually feasible as all dual edges are self-loops. We set the in-degree specification to 2 for the vertex at the intersection of the cycles and to 1 for the other vertices. Now, an orientation of the base graph is primally feasible, if and only if the edges of each cycle are all orientented in the same direction. As the two cycles can be oriented independently, the base graph has 4 feasible orientations. Examples of higher genus can be obtained by introducing $g$ copies of the embedding described above. The graphs are joined via an edge from node $b_{i}$ 
to $a_{i+1}$ for $i \in\{1, \ldots, g-1\}$. The resulting embedding has $5 g$ vertices and $7 g-1$ edges and still has only a single face. We increase the in-degree specifications of each base graph by setting $\alpha\left(a_{i+1}\right)=2$ for $i \in\{1, \ldots, g-1\}$, so that the new edges joining the copies have to be oriented from copy $i$ to copy $i+1$. The in-degree specification of the face is set to $|E|=7 g-1$. Now each copy of the base graph still has its 4 feasible orientations, so in total there are $4^{g}$ feasible orientations. $^{7}$

\section{Orientations with upper and lower bounds}

A generalization of the primal-dual orientation problem asks for an orientation that fulfills upper and lower bounds on the in-degrees of vertices and faces instead of attaining fixed values. We show that this problem becomes NP-complete, even when restricted to instances with embeddings of a fixed genus (e.g., planar graphs).

Problem 2. (Bounded primal-dual orientation problem)

Given: an embedded graph $G=(V, E)$, two pairs of functions $\alpha, \beta: V \rightarrow \mathbb{N}_{0}$ and $\alpha^{*}, \beta^{*}: V^{*} \rightarrow \mathbb{N}_{0}$

Task: Find an orientation $D$ of the edges $E$ such that $\alpha(v) \leq\left|\delta_{D}^{-}(v)\right| \leq \beta(v)$ for all $v \in V$ and $\alpha^{*}(f) \leq\left|\delta_{D}^{-}(f)\right| \leq \beta^{*}(f)$ for all $f \in V^{*}$, or prove that there is none.

Theorem 4. The bounded primal-dual orientation problem is NP-complete for graphs with any fixed genus.

Proof (Proof sketch). An orientation that solves the bounded primal-dual orientation problem can easily be verified in polynomial time. Hence, it remains to show that the problem is NP-hard. It is sufficient to do this for planar graphs. We use a reduction from planar 3-SAT, which is known to be an NP-hard problem [13]. We construct an instance of the bounded primal-dual orientation problem that has a solution if and only if the instance of planar 3-SAT has a solution. The construction consists of three main devices: For each variable, there is a variable gadget, for each clause, there is a clause gadget, and whenever a clause contains a variable, the corresponding gadgets are connected by an edge gadget. Figures 3 and 4 illustrate the construction. For a complete proof, please refer to the full version of this paper [4].

Corollary 5. The bounded primal-dual orientation problem is NP-complete even when restricted to instances with $\alpha=\beta$ or $\alpha^{*}=\beta^{*}$.

\footnotetext{
${ }^{7}$ Note that while the primal graph in the construction described above could also be embedded in a plane, this can be avoided by introducing additional vertices and edges.
} 

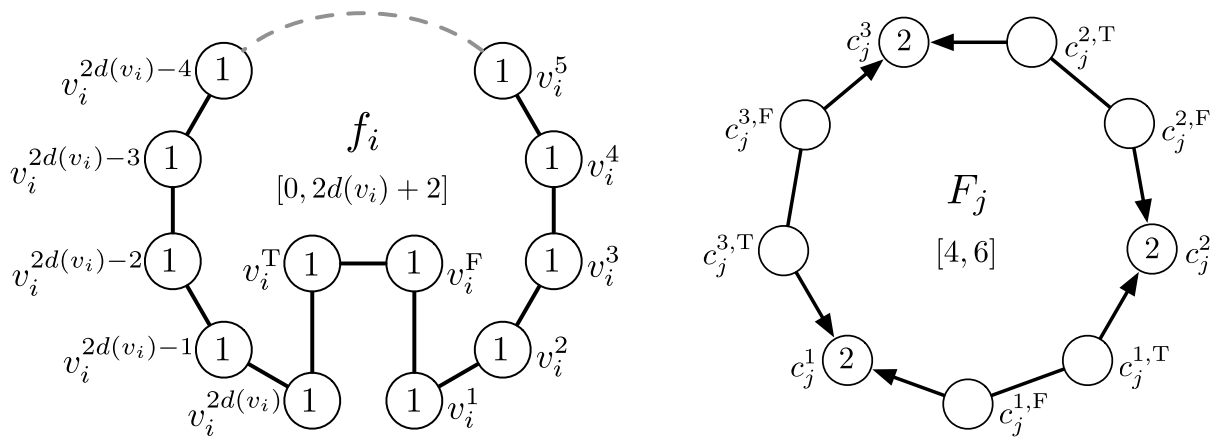

Fig. 3. Illustration of the variable gadget (left) and the clause gadget (right). The degree (number of occurrences) of variable $v_{i}$ is denoted by $d\left(v_{i}\right)$. The labels of the nodes correspond to their prescribed in-degrees, and the intervals on the faces correspond to a range of permitted in-degrees. The orientation of the edge $\left(v_{i}^{\mathrm{T}}, v_{i}^{\mathrm{F}}\right)$ corresponds to a truth assignment to variable $v_{i}$. The orientation of the edge $\left(c_{j}^{l, \mathrm{~T}}, c_{j}^{l, \mathrm{~F}}\right)$ corresponds to a truth assignment to literal $l$ in clause $C_{j}$.

Proof. This follows from the fact that the construction in the proof of Theorem 4 has $\alpha=\beta$. By duality, the reduction can also be achieved by an instance with $\alpha^{*}=\beta^{*}$.

\section{Conclusion}

We have shown that the primal-dual orientation problem in an embedded graph of genus $g$ has at most $2^{2 g}$ feasible solutions and the set of all solutions can be computed in time $\mathcal{O}\left(2^{2 g}|E|^{2}+|E|^{3}\right)$. In particular, the solution is unique if the embedding is planar. However, the problem becomes NP-hard immediately, if only upper and lower bounds on the in-degrees are specified.

While these results give a relatively clear characterization of the complexity of the primal-dual orientation problem, we still want to point out an open question resulting from our research: The algorithm proposed in the proof of Theorem 3 has a running time that is exponential in the genus of the embedding. Is it possible to devise an algorithm that finds a totally feasible orientation in time polynomial in the size of the graph and the genus of the embedding? In the case of the bounded primal-dual orientation problem, our results imply that parameterization by the genus did not have any effect on the complexity of the problem. This might be an indication that the primal-dual orientation problem with exact degree specifications is not only fixed-parameter tractable but actually solvable in polynomial time.

Acknowledgements. We thank Kristóf Bérczi and Júlia Pap for providing many helpful suggestions. This work has been supported by the Berlin Mathematical School and by Deutsche Forschungsgemeinschaft (DFG) as part of the Priority Program "Algorithm Engineering" (1307). 

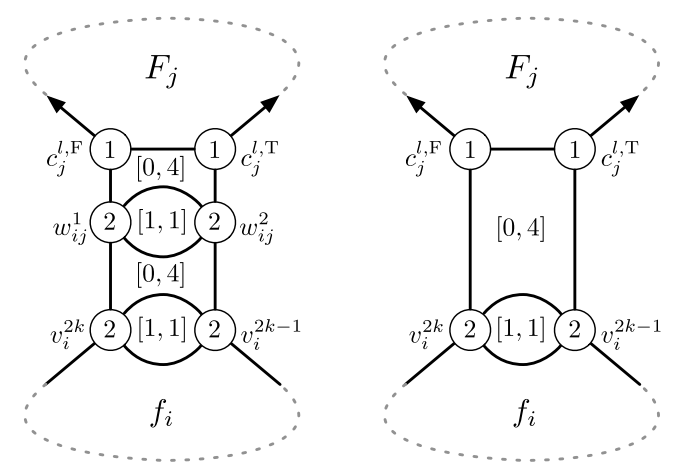

Fig. 4. Illustration of the edge gadget for an edge connecting variable $v_{i}$ with clause $C_{j}$. The gadget on the left is used when $v_{i}$ appears in a positive literal in $C_{j}$, and the one on the right is used when $v_{i}$ appears in a negative literal.

\section{References}

1. Y. Asahiro, J. Jansson, E. Miyano, and H. Ono. Upper and lower degree bounded graph orientation with minimum penalty. In Proceedings of the 18th Computing: The Australasian Theroy Symposium, to appear.

2. T. Biedl, T. Chan, Y. Ganjali, M.T. Hajiaghayi, and D.R. Wood. Balanced vertexorderings of graphs. Discrete Applied Mathematics, 148(1):27-48, 2005.

3. M. Chrobak and D. Eppstein. Planar orientations with low out-degree and compaction of adjacency matrices. Theoretical Computer Science, 86(2):243-266, 1991.

4. Y. Disser and J. Matuschke. Degree-constrained orientations of embedded graphs. Technical Report 032, TU Berlin, 2011.

5. P. Eades and N.C. Wormald. Fixed edge-length graph drawing is NP-hard. Discrete Applied Mathematics, 28(2):111-134, 1990.

6. J. Edmonds. Minimum partition of a matroid into independent subsets. Journal of Research National Bureau of Standards Section B, 69:67-72, 1965.

7. S. Felsner. Lattice structures from planar graphs. Journal of Combinatorics, 11(1):15, 2004.

8. A. Frank. Connections in combinatorial optimization. Oxford University Press, 2011.

9. A. Frank. personal communication, February 2010.

10. A. Frank and A. Gyárfás. How to orient the edges of a graph. Colloquia mathematica societatis Janos Bolyai, 18:353-364, 1976.

11. A. Frank, T. Király, and Z. Király. On the orientation of graphs and hypergraphs. Discrete Applied Mathematics, 131(2):385-400, 2003.

12. S.L. Hakimi. On the degrees of the vertices of a directed graph. Journal of the Franklin Institute, 279(4):290-308, 1965.

13. D. Lichtenstein. Planar formulae and their uses. SIAM Journal on Computing, 11(2):329-343, 1982.

14. H. Robbins. A theorem on graphs, with an application to a problem of traffic control. The American Mathematical Monthly, 46(5):281-283, 1939.

15. H. Whitney. Non-separable and planar graphs. Transactions of the American Mathematical Society, 34(2):339-362, 1932. 